\title{
Stage IA Uterine Corpus Endometrial Stromal Sarcoma AJCC v8
}

National Cancer Institute

\section{Source}

National Cancer Institute. Stage IA Uterine Corpus Endometrial Stromal Sarcoma A/CC v8. NCI Thesaurus. Code C139884.

Stage IA includes: T1a, N0, M0. T1a: Uterine corpus endometrial stroma sarcoma with tumor limited to the uterus, measuring $5 \mathrm{~cm}$ or less in greatest dimension. N0: No regional lymph node metastasis. M0: No distant metastasis. (AJCC 8th Ed.) 